\title{
Canagliflozin for the Treatment of Diabetic Kidney Disease and Implications for Clinical Practice: A Narrative Review
}

Davida Kruger · Virginia Valentine

Received: March 12, 2020 / Published online: May 13, 2020

(C) The Author(s) 2020

\section{ABSTRACT}

Type 2 diabetes mellitus (T2DM) affects millions of people worldwide, elevating their risk of developing a range of complications, including chronic kidney disease (CKD). People with T2DM and CKD (i.e., diabetic kidney disease, DKD) have an increased risk of progressing to end-stage kidney disease (ESKD), experiencing cardiovascular complications, and premature death. Despite this, DKD is primarily addressed through management of risk factors, and there are few pharmaceutical treatments capable of reversing or delaying disease progression. Canagliflozin is a sodium glucose co-transporter 2 inhibitor that was initially developed as

Digital Features To view enhanced digital features for this article go to https://doi.org/10.6084/m9.figshare. 12164172.

\section{Kruger $(\bowtie)$}

Henry Ford Health System, Detroit, MI, USA

e-mail: dkruger1@hfhs.org

V. Valentine

Clinica La Esperanza, Albuquerque, NM, USA a blood glucose-lowering agent for people with T2DM. Evidence from clinical trials of canagliflozin in people with T2DM, as well as evidence from cardiovascular outcomes trials in people with T2DM and high cardiovascular risk, provided preliminary evidence suggesting that it may also have beneficial renal effects. The Canagliflozin and Renal Events in Diabetes with Established Nephropathy Clinical Evaluation (CREDENCE) trial was a dedicated renal outcomes trial of canagliflozin that assessed its renal effects in people with DKD. Overall, the CREDENCE trial demonstrated that canagliflozin improves renal outcomes and slows early disease progression in people with DKD. These data supported the approval of canagliflozin for the treatment DKD, the first new treatment in almost 20 years; therefore, it is important for clinicians to understand how to implement this treatment in their clinical practice.

Keywords: Canagliflozin; Cardiovascular disease; Diabetic kidney disease; Randomized trials; Sodium glucose co-transporter 2 inhibitor; Type 2 diabetes 


\section{Key Summary Points}

People with type 2 diabetes have a high risk of developing diabetic kidney disease (DKD), which is the leading cause of endstage kidney disease

There are few pharmaceutical treatments capable of reversing or delaying the progression of DKD

The Canagliflozin and Renal Events in Diabetes with Established Nephropathy Clinical Evaluation (CREDENCE) trial was a dedicated renal outcomes trial of the sodium glucose co-transporter 2 inhibitor canagliflozin in people with DKD

Results from CREDENCE demonstrated that canagliflozin improves renal outcomes and slows early disease progression in people with DKD

This article reviews the key results from CREDENCE, along with context and guidance for clinicians on how to implement canagliflozin treatment in their clinical practice

\section{DIABETIC KIDNEY DISEASE: BURDEN AND TREATMENT}

More than 34 million Americans have diabetes, and over 90\% have type 2 diabetes mellitus (T2DM) [1, 2]. People with T2DM are at high risk of developing microvascular and macrovascular complications such as chronic kidney disease (CKD), which affects approximately $40 \%$ of people with T2DM and is the leading cause of end-stage kidney disease (ESKD) globally and in the USA [3-7]. CKD represents a variety of kidney-related diseases that are not necessarily related to T2DM; however, kidney disease that is pathologically related to T2DM can be classified as diabetic kidney disease (DKD). Clinically, DKD is identified by persistent urinary albumin to creatinine ratio
(UACR) $\geq 30 \mathrm{mg} / \mathrm{g}$ and/or sustained estimated glomerular filtration rate (eGFR) $<60 \mathrm{~mL} / \mathrm{min} /$ $1.73 \mathrm{~m}^{2}$ [8]. People diagnosed with DKD will experience continued reductions in eGFR, increased albuminuria, and glomerular hyperfiltration, all of which increase their cardiovascular risk [9-13]. DKD increases the rate of 10-year cumulative all-cause mortality in people with T2DM by almost $20 \%$, with the majority of this residual risk attributed to death from cardiovascular complications $[8,14]$. Heart failure is the most common cardiovascular complication among people with DKD, and the risk of developing heart failure is 2.5 times higher for people with T2DM compared to non-diabetic individuals, with this risk increasing with decreasing eGFR [15]. Osteoporosis, fracture, retinopathy, neuropathy, and amputation are also complications commonly seen in people with DKD [16-20].

Despite the risks associated with DKD, awareness of kidney disease is low among people with T2DM, particularly at early stages when the disease is generally asymptomatic; just $40-50 \%$ of people with stage 4 or 5 CKD are aware of their condition (Fig. 1) [21, 22]. The prevalence and major life-threatening risks associated with CKD make this condition costly for the health care system. In 2016, approximately $20 \%$ of overall Medicare expenditures were attributed to CKD (excluding ESKD), and more than $40 \%$ of Medicare spending in people with diabetes was allocated to the management of CKD [23]. The substantial impact of kidney disease led the US Department of Health and Human Services to announce its Advancing American Kidney Health initiative, which has a goal of reducing the number of Americans developing ESKD by $25 \%$ by 2020 [24].

Currently, the recommended approach to slow the progression of DKD is management of underlying risk factors, including hypertension, hypoxemia, hyperglycemia, albuminuria, and increased adiposity, along with refraining from smoking and following a low-protein diet $[9,25-28]$. Beyond this, people have few treatment options, and little progress has been made in this area over the past two decades, which has left a substantial proportion of people with DKD at high risk for progression to renal failure, 


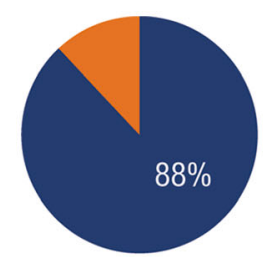

Any CKD

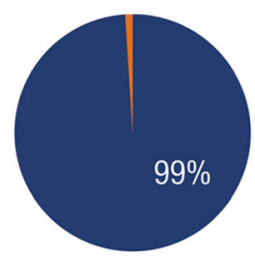

Stage 1

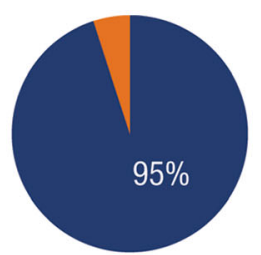

Stage 2

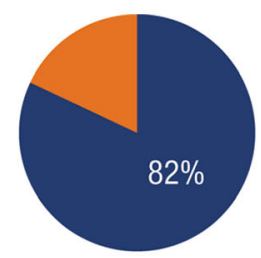

Stage 3

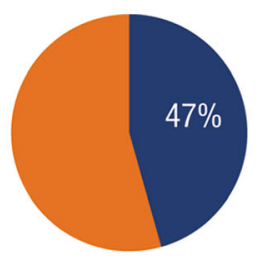

Stage 4

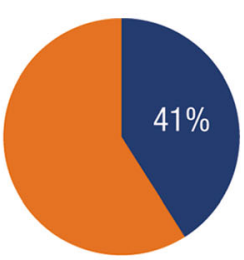

Stage 5

Undiagnosed Diagnosed

Fig. 1 Prevalence and awareness of CKD in adults with T2DM [11]. CKD chronic kidney disease, T2DM type 2 diabetes mellitus

cardiovascular morbidity, and cardiovascular mortality.

Renin-angiotensin-aldosterone system (RAAS) inhibitors, such as angiotensin-converting enzyme (ACE) inhibitors and/or angiotensin receptor blockers (ARBs), are recommended for the management of hypertension and heart failure [29-31]. Evidence from clinical trials in people with T2DM suggested that RAAS inhibitors may provide renal benefits in this population; however, only two ARBs (losartan and irbesartan) and no ACE inhibitors are approved for the prevention of kidney function decline in people with T2DM and albuminuria (> $300 \mathrm{mg} / \mathrm{g}) \quad[29,30,32-35]$. Additionally, RAAS inhibitors have been associated with safety risks such as hyperkalemia and acute kidney injury $[9,26]$. Considering the current state of treatment for DKD, new medications capable of preventing or delaying the progression of this disease are needed.

Recent studies have investigated the potential renal benefits associated with sodium glucose co-transporter 2 (SGLT2) inhibitors, which were first developed and approved for the management of hyperglycemia in people with T2DM [36-38]. Unlike other classes of antihyperglycemic agents (AHAs), SGLT2 inhibitors work independently of insulin by lowering the renal threshold for glucose excretion $\left(\mathrm{RT}_{\mathrm{G}}\right)$ in people with T2DM, thereby increasing urinary glucose excretion (UGE) and leading to a mild osmotic diuresis and a net caloric loss that contribute to improvements in glycemic control, weight loss, and blood pressure reduction $[39,40]$. SGLT2 inhibition has also been associated with renal hemodynamic changes, such as attenuation of renal hyperfiltration and normalization of tubuloglomerular feedback, which may contribute to renal benefits (Fig. 2) [41].

Evidence from cardiovascular outcome trials (CVOTs) of SGLT2 inhibitors in people with T2DM and established cardiovascular disease or cardiovascular risk provided evidence suggesting that this class may also have beneficial renal effects [42-44]. However, the patient populations enrolled in the CVOTs were generally at low renal risk, with eGFR ranging from 60 to $<90 \mathrm{~mL} / \mathrm{min} / 1.73 \mathrm{~m}^{2}$ and UACR $<30 \mathrm{mg} / \mathrm{g}$. In addition, the number of events accumulated was not enough to definitively establish the effects on renal outcomes [43]. For example, in the CANagliflozin cardioVascular Assessment Study (CANVAS) Program, which was the CVOT for the SGLT2 inhibitor canagliflozin, participants had mean eGFR of $76.5 \mathrm{~mL} / \mathrm{min} / 1.73 \mathrm{~m}^{2}$, median UACR of $12.3 \mathrm{mg} / \mathrm{g}$, and $80.0 \%$ were receiving a RAAS inhibitor [43]. CVOTs for other SGLT2 inhibitors, including EMPA-REG OUTCOME (empagliflozin) and DECLARETIMI 58 (dapagliflozin), recruited a similar population with low renal risk. As a result of this population profile, the number of renal outcomes accumulated in these trials was too low to definitively determine the impact of SGLT2 inhibitors on renal outcomes; there were only 69 confirmed renal replacement therapy or renal death events across the three studies, which included more than 34,000 participants [42-44]. Additionally, these studies were not designed to specifically assess the impact of intervention on renal endpoints, so all renal studies were based on secondary, exploratory, 


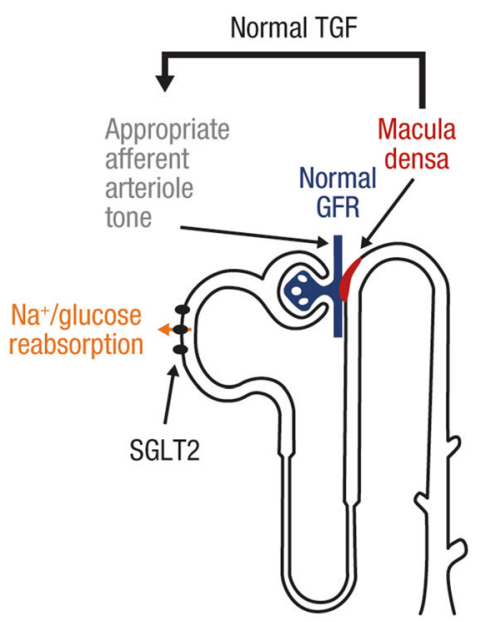

Normal physiology

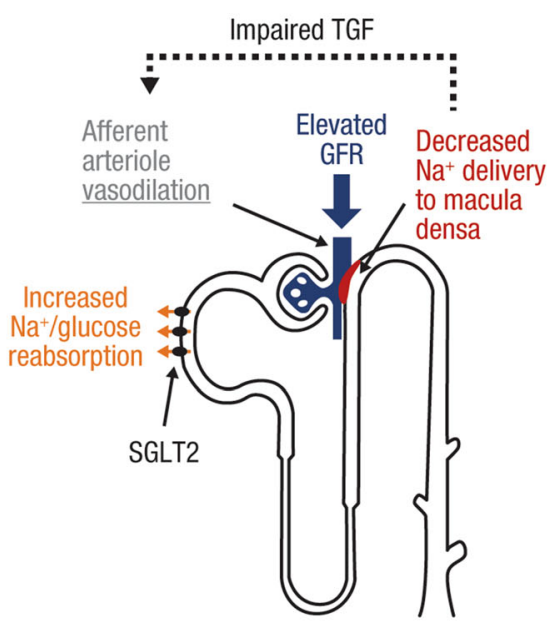

Hyperfiltration in early stages of diabetic nephropathy

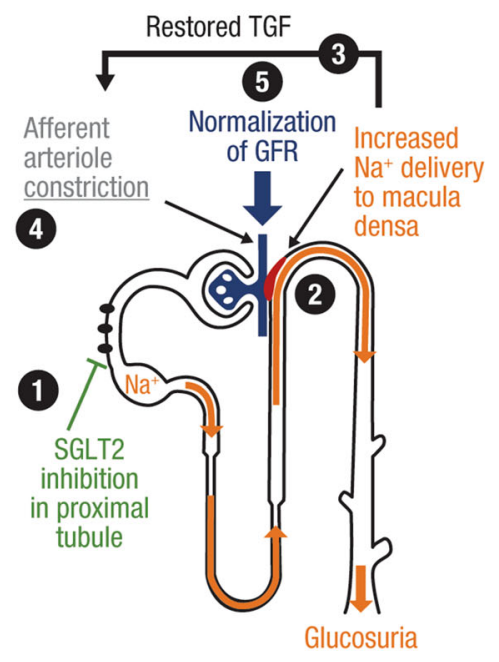

SGLT2 inhibition reduces hyperfiltration via TGF
Fig. 2 Tubuloglomerular feedback mechanisms encountered under normal physiology, in the early stages of diabetic nephropathy, and after SGLT2 inhibition [41]. SGLT2 sodium glucose co-transporter 2, TGF

or post hoc analyses. Thus, dedicated trials of SGLT2 inhibitors in people with DKD were necessary to assess the renal benefits afforded through treatment of this population with this class of medications. This article describes the dedicated renal outcomes trial, Canagliflozin and Renal Events in Diabetes with Established Nephropathy Clinical Evaluation (CREDENCE), which was the first study to demonstrate the renal effects of canagliflozin in people with T2DM and compromised kidney function (i.e., DKD) [45].

\section{COMPLIANCE WITH ETHICS GUIDELINES}

This article is based on previously conducted studies and does not contain any studies with human participants or animals performed by any of the authors.

\section{CREDENCE STUDY DESIGN}

CREDENCE was an event-driven trial designed to formally test whether canagliflozin lowers tubuloglomerular feedback, GFR glomerular filtration rate. Reproduced with permission from Cherney et al. [41]

the risk of kidney failure and cardiovascular outcomes in people with T2DM and established diabetic nephropathy $[45,46]$. The CREDENCE trial was initiated in 2014 before the conclusion of the CVOTs and its initiation was motivated by renal data measured in phase 3 studies of canagliflozin [45]. The study population included adults at least 30 years of age with T2DM $(\mathrm{HbA} 1 \mathrm{c} \geq 6.5 \%$ and $\leq 12.0 \%)$, eGFR $\geq 30$ and $<90 \mathrm{~mL} / \mathrm{min} / 1.73 \mathrm{~m}^{2}$, and UACR $>300$ and $\leq 5000 \mathrm{mg} / \mathrm{g}[45,46]$. People were also required to be receiving a maximally tolerated dose of an ACE inhibitor or ARB for at least 4 weeks prior to randomization $[45,46]$. The CREDENCE trial was stopped 2 years early on the advice of an independent data monitoring committee on the basis of the efficacy and safety findings observed in an interim analysis conducted in July 2018 after a planned 405 primary endpoint events had occurred [46].

The primary endpoint of the CREDENCE trial was the composite of ESKD (defined as the need for chronic dialysis for 30 days or more, kidney transplantation, or eGFR $<15 \mathrm{~mL} / \mathrm{min} /$ $1.73 \mathrm{~m}^{2}$ sustained for at least 30 days by central laboratory assessment), doubling of serum 
creatinine, or renal or cardiovascular death $[45,46]$. Secondary endpoints were tested according to a prespecified hierarchical testing sequence as follows: cardiovascular death or hospitalization for heart failure; cardiovascular death, myocardial infarction, or stroke (i.e., 3-point major adverse cardiovascular event, MACE); hospitalization for heart failure; the composite of ESKD, doubling of serum creatinine, or renal death; cardiovascular death; allcause mortality; and the composite of cardiovascular death, myocardial infarction, stroke, hospitalization for heart failure, or hospitalization for unstable angina $[45,46]$. All renal and cardiovascular endpoints that were part of planned primary and secondary composite endpoints were adjudicated by a blinded endpoint adjudication committee $[45,46]$. Other outcomes of interest included the effects on intermediate outcomes, including eGFR, UACR, HbA1c, and blood pressure.

\section{EFFICACY OUTCOMES WITH CANAGLIFLOZIN IN CREDENCE}

A total of 4401 people were enrolled in the CREDENCE trial and randomized (1:1) to canagliflozin $100 \mathrm{mg}$ or placebo. Participants had a mean age of 63 years, HbA1c of $8.3 \%$, body mass index of $31.3 \mathrm{~kg} / \mathrm{m}^{2}$, and had T2DM for an average duration of 16 years. Most CREDENCE trial participants were male $(66 \%)$ and white (67\%) [46]. In addition, $60 \%$ of people had eGFR $<60 \mathrm{~mL} / \mathrm{min} / 1.73 \mathrm{~m}^{2}$ at baseline. Overall, participants had a mean eGFR of $56.2 \mathrm{~mL} /$ $\mathrm{min} / 1.73 \mathrm{~m}^{2}$, a median UACR of $927 \mathrm{mg} / \mathrm{g}$, and 99.9\% were taking a maximally tolerated dose of a RAAS inhibitor.

Canagliflozin treatment was associated with a $30 \%$ relative risk reduction of the primary composite outcome of ESKD, doubling of serum creatinine, or renal or cardiovascular death compared with placebo (hazard ratio [HR], 0.70; 95\% confidence interval [CI] 0.59-0.82; $P=0.00001$; Fig. 3) [46]. Consistent effects were seen for the components of the primary composite endpoint, as well as the secondary renal, cardiovascular, and mortality endpoints (Fig. 4).
The effects of canagliflozin on reducing the risk of the primary outcome were observed consistently across participant subgroups, including those defined by history of cardiovascular disease, screening eGFR, race, ethnicity, and baseline HbA1c; effects were also generally similar across subgroups for other renal, cardiovascular, and mortality outcomes [47-50]. Of note, canagliflozin was the first AHA to demonstrate renal and cardiovascular benefits in a population without cardiovascular disease history [50]. The renal and cardiovascular effects of canagliflozin were also consistent across eGFR subgroups, including in participants with screening eGFR of 30 to $<45 \mathrm{~mL} /$ $\mathrm{min} / 1.73 \mathrm{~m}^{2}[48,51]$. A post hoc analysis in participants with baseline eGFR $<30 \mathrm{~mL} / \mathrm{min} /$ $1.73 \mathrm{~m}^{2}(n=174)$ also demonstrated consistent effects of canagliflozin on renal, cardiovascular, and mortality outcomes as those seen in the overall population, with no differences in the risk of renal-related adverse events or other safety outcomes compared to the overall population $[46,48]$.

As seen in previous studies, canagliflozin treatment was associated with an acute drop in eGFR from baseline to week 3 (Fig. 5) [46]. This initial decline in eGFR was seen rapidly and attenuated over time [52]. After this initial drop, the rate of eGFR decline was reduced by approximately $60 \%$ with canagliflozin compared to placebo, suggesting that canagliflozin treatment may help to delay progression of renal function decline [46]. In addition, canagliflozin was also associated with a $31 \%$ reduction in UACR compared with placebo over the course of the trial.

The number needed to treat (NNT) has been reported for key outcomes from the CREDENCE trial (Fig. 6). The NNT to prevent one event from the primary composite outcome was 22 (95\% CI 15-38). NNTs for other outcomes were 28 (95\% CI 19-54) for the composite renal outcomes of ESKD, doubling of the serum creatinine level, or renal death; $43(95 \%$ CI 26-121) for ESKD events; and 46 (95\% CI 29-124) for hospitalization for heart failure. These findings further demonstrate the beneficial effects of canagliflozin on renal and cardiovascular outcomes in people with DKD. 


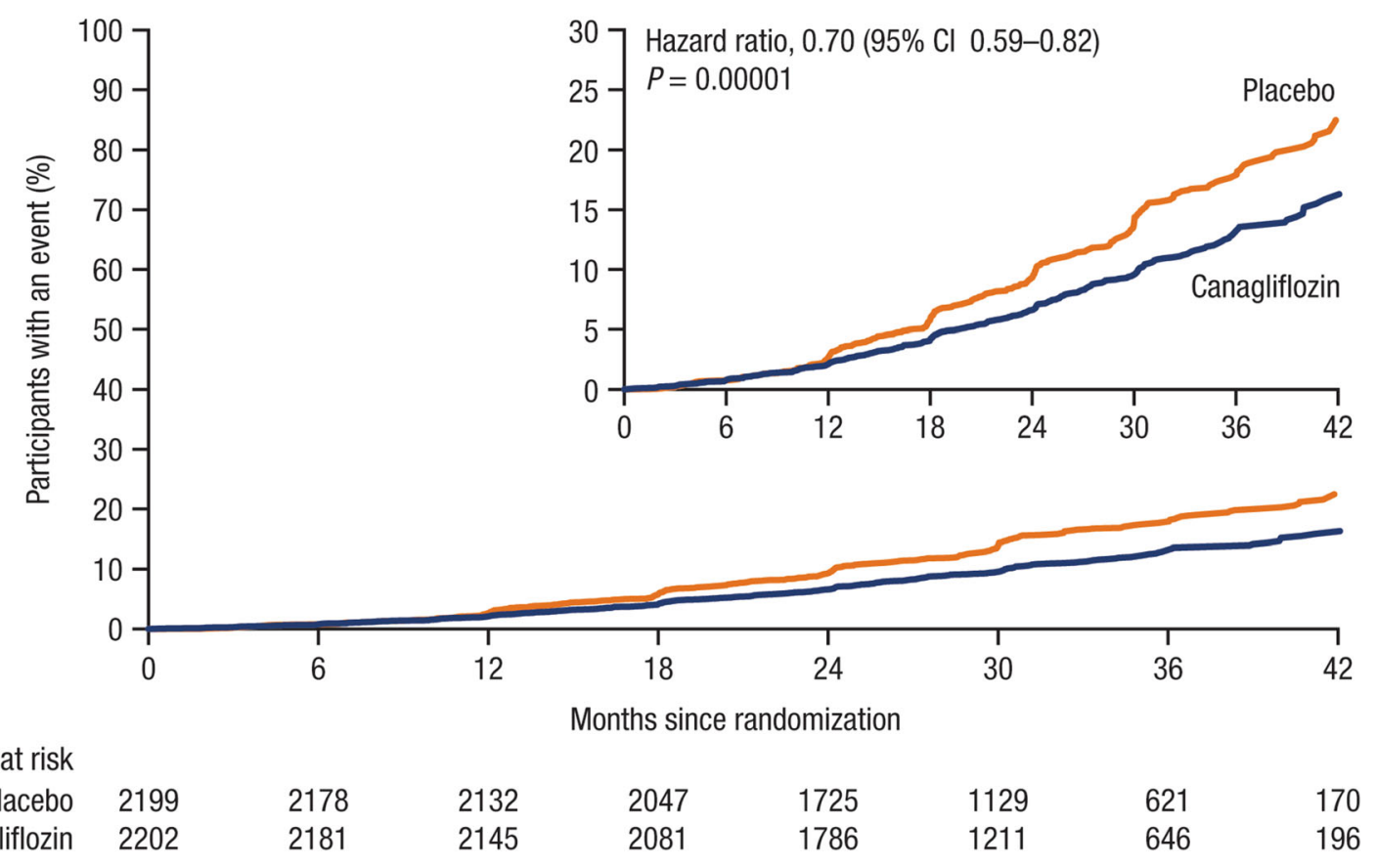

Fig. 3 Primary composite outcome of ESKD, doubling of serum creatinine, or renal or cardiovascular death in CREDENCE [46]. ESKD end-stage kidney disease. From

\section{SAFETY OUTCOMES WITH CANAGLIFLOZIN IN CREDENCE}

The overall safety profile of canagliflozin in the CREDENCE trial was generally consistent with the previous studies of canagliflozin (Fig. 7) [46]. In CREDENCE, overall adverse events and serious adverse events were lower among participants receiving canagliflozin compared with placebo. There was no difference between canagliflozin and placebo for the incidence of adverse events of interest, including malignancies, urinary tract infections, volume depletion, hypoglycemia, osmotic diuresis, and hypersensitivity/cutaneous reactions [46]. Furthermore, canagliflozin treatment resulted in a lower risk of renal-related adverse events compared to those treated with placebo (HR, 0.71; 95\% CI 0.61-0.82) [46]. There was no difference observed in the risk of acute kidney injury between the participants receiving canagliflozin and placebo (HR, 0.85; 95\% CI 0.64-1.13) [46].
Perkovic et al. [46]. Copyright (C) 2020 Massachusetts Medical Society. Reprinted with permission from Massachusetts Medical Society

Notably, in CREDENCE there was no difference between canagliflozin and placebo in the rates of amputation (HR, 1.11; 95\% CI $0.79-1.56)$ or fracture (HR, 0.98; 95\% CI $0.70-1.37$ ) [46]. These results are in contrast with the CANVAS Program, which found an increased risk of amputation (HR, 1.97; 95\% CI 1.41-2.75), and the CANVAS trial, which found an increased risk of fracture (HR, 1.55; 95\% CI 1.21-1.97) in participants treated with canagliflozin [43]. The reason for this discrepancy is not known and, with the exception of an increased risk of amputation observed in studies of ertugliflozin, other clinical studies of SGLT2 inhibitors, including phase 3 trials of canagliflozin, have not shown this same risk [44, 53-55].

\section{CLINICAL PERSPECTIVE}

DKD is a devastating disease that puts people with T2DM at an increased risk for developing several serious and life-threatening conditions. The dearth of new treatments for DKD over the 


\begin{tabular}{|c|c|c|c|c|c|}
\hline & \multicolumn{2}{|c|}{$\begin{array}{l}\text { Number of participants } \\
\text { with an event }\end{array}$} & \multirow[b]{2}{*}{$\begin{array}{l}\text { Hazard ratio } \\
(95 \% \mathrm{Cl})\end{array}$} & & \multirow[b]{2}{*}{$P$ value } \\
\hline & $\begin{array}{l}\text { Canagliflozin } \\
\text { (N = 2202) }\end{array}$ & $\begin{array}{l}\text { Placebo } \\
(\mathrm{N}=2199)\end{array}$ & & & \\
\hline Primary composite outcome* & 245 & 340 & 터 & $0.70(0.59-0.82)$ & 0.00001 \\
\hline Doubling of serum creatinine & 118 & 188 & $10-1$ & $0.60(0.48-0.76)$ & $<0.001$ \\
\hline End-stage kidney disease & 116 & 165 & $1 \bullet-1 !$ & $0.68(0.54-0.86)$ & 0.002 \\
\hline $\mathrm{eGFR}<15 \mathrm{~mL} / \mathrm{min} / 1.73 \mathrm{~m}^{2}$ & 78 & 125 & $\mapsto-1 !$ & $0.60(0.45-0.80)$ & - \\
\hline Dialysis initiated or kidney transplantation & 76 & 100 & $\mapsto-1$ & $0.74(0.55-1.00)$ & - \\
\hline Renal death & 2 & 5 & 1 & $0.39(0.08-2.03)$ & - \\
\hline CV death & 110 & 140 & $10-1$ & $0.78(0.61-1.00)$ & 0.0502 \\
\hline CV death or hospitalization for heart failure & 179 & 253 & re- i & $0.69(0.57-0.83)$ & $<0.001$ \\
\hline CV death, myocardial infarction, or stroke & 217 & 269 & 10-1 & $0.80(0.67-0.95)$ & 0.01 \\
\hline Hospitalization for heart failure & 89 & 141 & $10-1$ & $0.61(0.47-0.80)$ & $<0.001$ \\
\hline All-cause mortality & 168 & 201 & $10-1$ & $0.83(0.68-1.02)$ & - \\
\hline $\begin{array}{l}\text { CV death, myocardial infarction, stroke, or } \\
\text { hospitalization for heart failure or unstable angina }\end{array}$ & 273 & 361 & rer 1 & $0.74(0.63-0.86)$ & - \\
\hline $\begin{array}{l}\text { End-stage kidney disease, doubling of serum creatinine, } \\
\text { or renal death }\end{array}$ & 153 & 224 & He- i & $0.66(0.53-0.81)$ & $<0.001$ \\
\hline Dialysis, kidney transplantation, or renal death ${ }^{\dagger}$ & 78 & 105 & $\mapsto-i$ & $0.72(0.54-0.97)$ & - \\
\hline & & 0. & 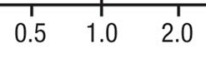 & 4.0 & \\
\hline & & & $\begin{array}{ll}\text { Favors } & \text { Favors } \\
\text { gliflozin } & \text { placebo }\end{array}$ & & \\
\hline
\end{tabular}

Fig. 4 Effects of canagliflozin on cardiovascular and renal outcomes in CREDENCE [46]. CI confidence interval, eGFR estimated glomerular filtration rate, $\mathrm{CV}$

last two decades has presented a challenge for disease management and created an immense economic burden. The CREDENCE trial was uniquely designed compared with previous trials because of its focus on renal outcomes and its study population, which included people with DKD. Thus, CREDENCE was the first study to provide definitive evidence demonstrating the effects of canagliflozin in slowing kidney function decline in this population with compromised kidney function. In addition to its renal benefits, CREDENCE was the first study to show the efficacy of an AHA for preventing cardiovascular events in those without a history of cardiovascular disease [46].

In addition to providing evidence for the beneficial effects of canagliflozin in slowing kidney function decline in people with DKD, results from the CREDENCE trial demonstrated a favorable safety profile that did not result in an increased risk of amputation or fracture with canagliflozin. It remains important for cardiovascular. ${ }^{*}$ The primary composite outcome included end-stage kidney disease, doubling of serum creatinine, or renal or $\mathrm{CV}$ death. ${ }^{\dagger}$ This outcome was exploratory

clinicians to remember that good clinical practice and proper foot care are always crucial in people with DKD, who are at an inherently heightened baseline risk for amputation and fracture.

The mechanism responsible for the renal benefits associated with canagliflozin treatment in the CREDENCE trial is unclear; however, a number of potential hypotheses have been generated. It has been proposed that the renal benefits associated with SGLT2 inhibition are achieved through a renal hemodynamic effect that may attenuate renal hyperfiltration and normalization of tubuloglomerular feedback by blocking sodium reabsorption at renal proximal tubules, ultimately increasing sodium delivery to the macula densa [41, 56-59]. A number of alternative mechanisms have been proposed and are under investigation, including effects on albuminuria; glucose-mediated inflammation, proliferation, and fibrosis; proximal tubule hypertrophy; oxidative stress; and 


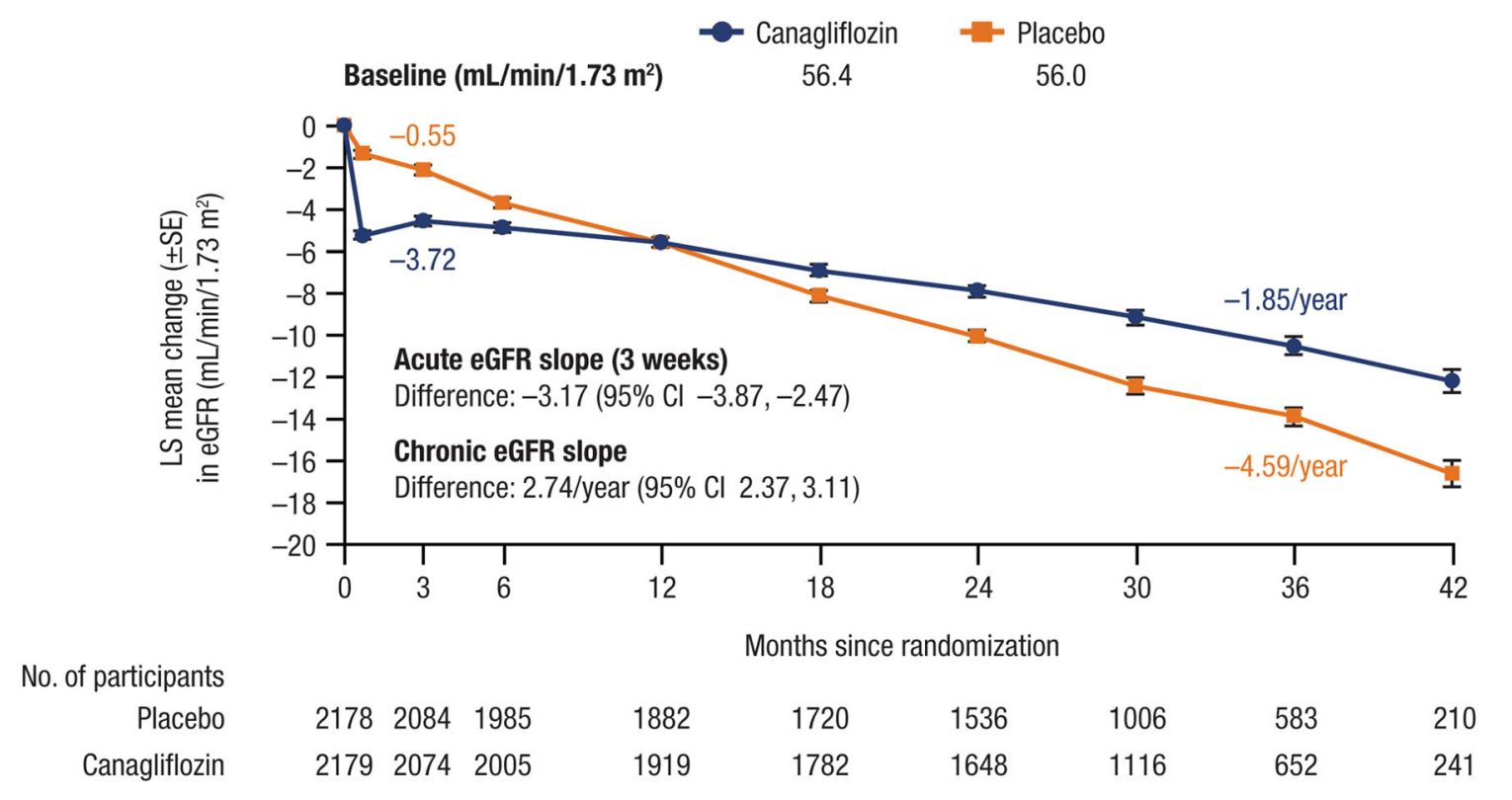

Fig. 5 Effects of canagliflozin on eGFR slope over time [46]. eGFR estimated glomerular filtration rate, LS least squares, SE standard error, CI confidence interval. From

Perkovic et al. [46] Copyright (C) 2020 Massachusetts Medical Society. Reprinted with permission from Massachusetts Medical Society

\section{A Primary composite outcome*}

\section{2 Tiा}

\section{End-stage kidney disease}

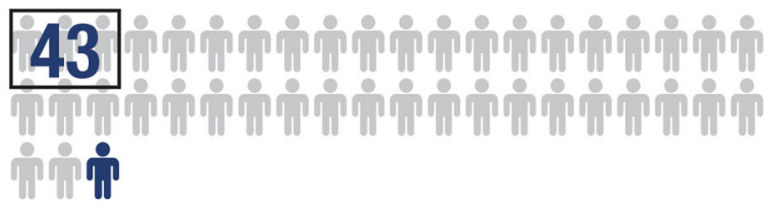

Fig. 6 Number needed to treat to prevent one event from the a primary composite outcome; $\mathbf{b}$ composite renal outcomes of end-stage kidney disease, doubling of the serum creatinine level, or renal death; c end-stage kidney disease; d hospitalization for heart failure [46]. eGFR estimated glomerular filtration rate. ${ }^{*}$ ESKD (defined as the

sodium-hydrogen exchanger expression [60-64]. Furthermore, it has been hypothesized that SGLT2 inhibitors complement the mechanism of RAAS inhibition to improve renal and

\section{B End-stage kidney disease, doubling of serum creatinine, or renal death}

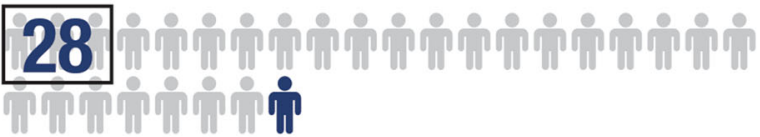

\section{Hospitalization for heart failure}

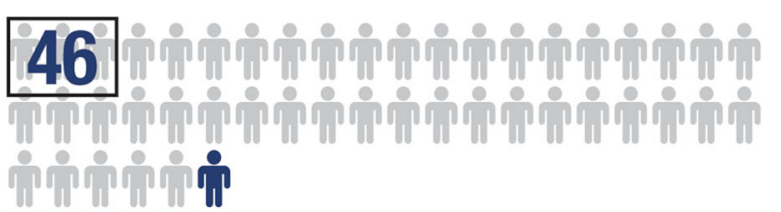

need for chronic dialysis for at least 30 days, kidney transplantation, or eGFR $<15 \mathrm{~mL} / \mathrm{min} / 1.73 \mathrm{~m}^{2}$ sustained for at least 30 days by central laboratory assessment), doubling of serum creatinine, or renal or cardiovascular death

cardiovascular outcomes in people receiving ACE inhibitors and/or ARBs [65]. Indeed, the renal benefits associated with canagliflozin treatment were observed in addition to standard 


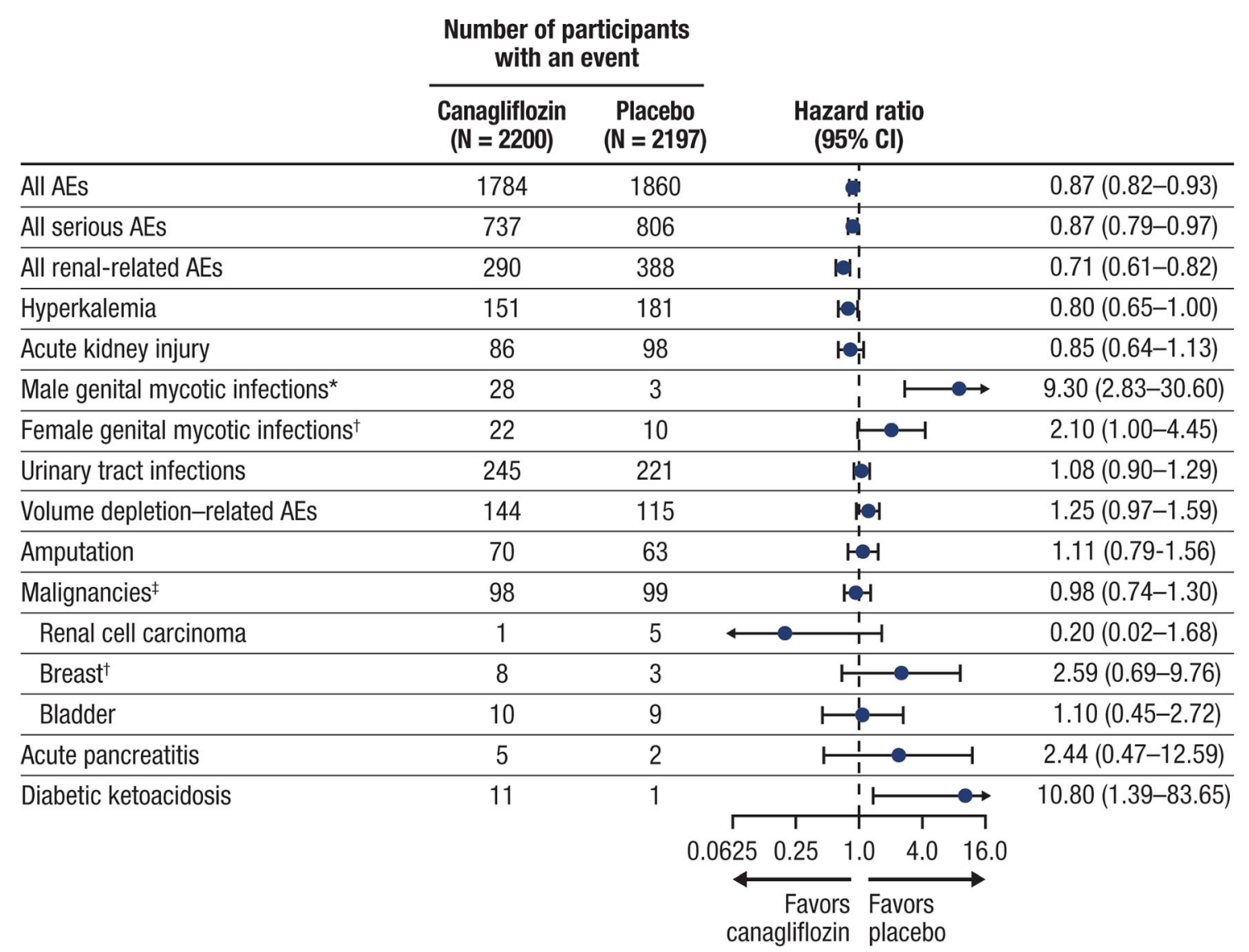

Fig. 7 Effects of canagliflozin on safety outcomes in CREDENCE [46]. CI confidence interval, AE adverse event. ${ }^{*}$ Includes male participants only (canagliflozin,

of care with RAAS inhibitors, which were being taken by more than $99 \%$ of CREDENCE trial participants. Future studies will be helpful to decipher the mechanism responsible for the renal benefits observed with canagliflozin, which may help to inform future prescribing recommendations related to the combined use of SGLT2 inhibitors and RAAS inhibitors.

Renal outcomes data for people with DKD are not yet available for other SGLT2 inhibitors; however, trials for the SGLT2 inhibitors dapagliflozin and empagliflozin that include this population as well as people with CKD (without T2DM) are ongoing and expected to be completed in late 2020 and 2022, respectively $[66,67]$. These data may help to clarify whether the renal benefits seen with canagliflozin in CREDENCE trial participants are a class effect of $n=1439$; placebo, $n=1466)$. ${ }^{\dagger}$ Includes female participants only (canagliflozin, $n=761$; placebo, $n=731$ ).

*ncludes malignant tumors of unspecified type

SGLT2 inhibitors and whether these effects may extend to people with CKD who do not have T2DM to further establish the renal efficacy and safety of this class of drugs.

The results from the CREDENCE trial prompted the US Food and Drug Administration (FDA) to expand the indication for canagliflozin to a new patient population [68]. Prior to CREDENCE, canagliflozin was approved for two indications: (1) as an adjunct to diet and exercise to improve glycemic control in adults with T2DM and (2) to reduce the risk of MACE in adults with T2DM and established cardiovascular disease. On the basis of results from the CREDENCE trial, the FDA approved canagliflozin to reduce the risk of ESKD, doubling of serum creatinine, cardiovascular death, and hospitalization for heart failure in adults with 


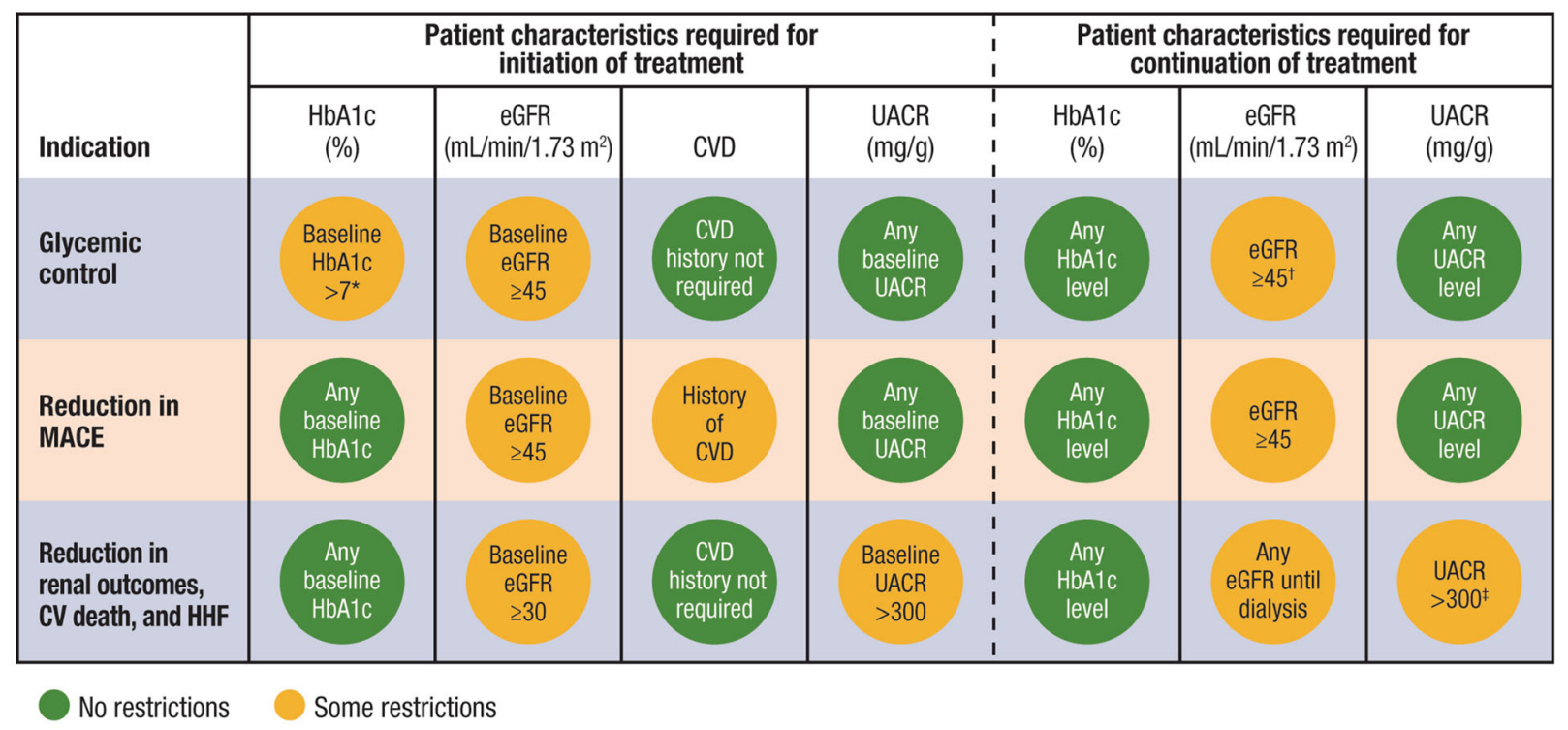

Fig. 8 Updated dosing and eligibility guidelines for canagliflozin [68]. eGFR estimated glomerular filtration rate, CVD cardiovascular disease, UACR urinary albumin to creatinine ratio, MACE major adverse cardiovascular events, CV cardiovascular, HHF hospitalization for heart failure. Note that "No restrictions" and "Some restrictions" refer to the eligibility requirements of patients for treatment with canagliflozin based on the criteria specified

T2DM and diabetic nephropathy with albuminuria (> $300 \mathrm{mg} /$ day). The US prescribing information for canagliflozin was also updated to allow the continuation of canagliflozin $100 \mathrm{mg}$ in people already receiving therapy whose eGFR falls to $<30 \mathrm{~mL} / \mathrm{min} / 1.73 \mathrm{~m}^{2}$ with a UACR $>300 \mathrm{mg} / \mathrm{g}$ until initiation of dialysis or kidney transplantation [68]. Evidence from the CREDENCE trial also prompted the American Diabetes Association to update its guidelines in June 2019 to recommend that people with T2DM with an eGFR $\geq 30 \mathrm{~mL} / \mathrm{min} /$ $1.73 \mathrm{~m}^{2}$ and albuminuria ( $>300 \mathrm{mg} / \mathrm{g}$ ) consider using an SGLT2 inhibitor to reduce their risk of DKD progression and/or cardiovascular events [69]. Further updates to these guidelines published in 2020 suggested that SGLT2 inhibitors should be considered independently of HbA1c for people when atherosclerotic cardiovascular disease, heart failure, or CKD predominates or for people with T2DM and CKD who require treatment in addition to metformin to attain in each row/column. For those with "Some restrictions," the specific thresholds are provided in the figure. ${ }^{*}$ Varies depending on specific goals of patient. ${ }^{\dagger}$ Dose can be increased to $300 \mathrm{mg}$ for better glycemic control if eGFR $>60 \mathrm{~mL} / \mathrm{min} / 1.73 \mathrm{~m}^{2}$. \$Patient can continue treatment with eGFR $<30 \mathrm{~mL} / \mathrm{min} / 1.73 \mathrm{~m}^{2}$ as long as $\mathrm{UACR}$ is $>300 \mathrm{mg} / \mathrm{g}$

target HbA1c [70]. Taken together, these new recommendations demonstrate that canagliflozin treatment is beneficial to a diverse array of people in a broad range of circumstances, and physicians should consider the unique characteristics of each patient when determining if canagliflozin is an appropriate treatment option (Fig. 8).

\section{CONCLUSION}

The CREDENCE trial has demonstrated the effects of canagliflozin that improve renal outcomes and slow early disease progression in people with T2DM and DKD. In addition to the positive effects on kidney function, treatment with canagliflozin also decreased the prevalence of all comorbid complications in CREDENCE trial participants, demonstrating the potential this treatment has to reduce the DKD-related expenditures in the health care industry. Data from other trials investigating the use of SGLT2 
inhibitors will be crucial for further establishing the value and efficacy of this class of drugs in people at high renal risk and probing the extension of these benefits to a wider range of people.

\section{ACKNOWLEDGEMENTS}

Funding. This article and the fee for the journal's Rapid Service were supported by Janssen Scientific Affairs, LLC (Titusville, NJ, USA). The authors did not receive financial compensation for their contributions to this article.

Authorship. All named authors meet the International Committee of Medical Journal Editors (ICMJE) criteria for authorship for this article, take responsibility for the integrity of the work as a whole, and have given their approval for this version to be published. Both authors contributed to the conception, drafting, and revision of the article and approved the final version for submission.

Medical Writing, Editorial, and Other Assistance. Medical writing support was provided by Elizabeth Meucci, PhD, of MedErgy, and was funded by Janssen Scientific Affairs, LLC.

Disclosures. Davida Kruger has served as an advisor for Novo Nordisk, Abbott, Sanofi Aventis, Janssen, Dexcom, Merck, Pendulum, Boehringer Ingelheim, and Lilly and as a speaker for Janssen, Valeritas, AstraZeneca, Boehringer Ingelheim, Lilly, Novo Nordisk, Dexcom, Abbott, and Insulet. She reports receiving grants or research funding from Novo Nordisk, the Hemsley Foundation, Dexcom, Lexicon, and Abbott and owns stock in Pendulum. Virginia Valentine has served as an advisor for Janssen, AstraZeneca, Boehringer Ingelheim, Merck, Lilly, Lexicon, Abbott Diabetes Care, Novo Nordisk, and Sanofi and as a speaker for Lilly and Boehringer Ingelheim.

Compliance with Ethics Guidelines. This article is based on previously conducted studies and does not contain any studies with human participants or animals performed by any of the authors.

Data Availability. Data sharing is not applicable to this article as no datasets were generated or analyzed during the current study.

Open Access. This article is licensed under a Creative Commons Attribution-NonCommercial 4.0 International License, which permits any non-commercial use, sharing, adaptation, distribution and reproduction in any medium or format, as long as you give appropriate credit to the original author(s) and the source, provide a link to the Creative Commons licence, and indicate if changes were made. The images or other third party material in this article are included in the article's Creative Commons licence, unless indicated otherwise in a credit line to the material. If material is not included in the article's Creative Commons licence and your intended use is not permitted by statutory regulation or exceeds the permitted use, you will need to obtain permission directly from the copyright holder. To view a copy of this licence, visit http://creativecommons.org/licenses/by$\mathrm{nc} / 4.0 /$.

\section{REFERENCES}

1. DeFronzo RA. Pathogenesis of type 2 diabetes mellitus. Med Clin N Am. 2004;88(787-835):ix.

2. National Center for Chronic Disease Prevention and Health Promotion. National diabetes statistics report, 2020. Estimates of diabetes and its burden in the United States. 2020. https://www.cdc.gov/ diabetes/pdfs/data/statistics/national-diabetesstatistics-report.pdf. Accessed 20 Feb 2020.

3. System USRD. Chapter 1: CKD in the general population. In: 2018 Annual Report. https:/www. usrds.org/2018/download/v1_c01_GenPop_18_ usrds.pdf. Accessed 20 Feb 2020.

4. Centers for Disease Control and Prevention. National chronic kidney disease fact sheet, 2017. 2017. https://www.cdc.gov/diabetes/pubs/pdf/ kidney_factsheet.pdf. Accessed 20 Feb 2020. 
5. United States Renal Data System. 2017 USRDS annual data report: epidemiology of kidney disease in the United States. 2017. https://www.usrds.org/ 2017/view/Default.aspx. Accessed 20 Feb 2020.

6. Cameron JS. The discovery of diabetic nephropathy: from small print to centre stage. J Nephrol. 2006;19:S75-87.

7. Koye DN, Magliano DJ, Nelson RG, Pavkov ME. The global epidemiology of diabetes and kidney disease. Adv Chronic Kidney Dis. 2018;25:121-32.

8. Alicic RZ, Rooney MT, Tuttle KR. Diabetic kidney disease: challenges, progress, and possibilities. Clin J Am Soc Nephrol. 2017;12:2032-45.

9. Tuttle KR, Bakris GL, Bilous RW, et al. Diabetic kidney disease: a report from an ADA Consensus Conference. Diabetes Care. 2014;37:2864-83.

10. Reboldi G, Verdecchia P, Fiorucci G, et al. Glomerular hyperfiltration is a predictor of adverse cardiovascular outcomes. Kidney Int. 2018;93: 195-203.

11. Gansevoort RT, Correa-Rotter R, Hemmelgarn BR, et al. Chronic kidney disease and cardiovascular risk: epidemiology, mechanisms, and prevention. Lancet. 2013;382:339-52.

12. Ruggenenti P, Porrini EL, Gaspari F, et al. Glomerular hyperfiltration and renal disease progression in type 2 diabetes. Diabetes Care. 2012;35: 2061-8.

13. Tancredi M, Rosengren A, Svensson AM, et al. Excess mortality among persons with type 2 diabetes. N Engl J Med. 2015;373:1720-32.

14. Afkarian M, Sachs M, Kestenbaum B, et al. Kidney disease and increased mortality risk in type 2 diabetes. J Am Soc Nephrol. 2013;24:302-8.

15. Tancredi M, Rosengren A, Olsson $M$, et al. The relationship between three eGFR formulas and hospitalization for heart failure in 54486 individuals with type 2 diabetes. Diabetes Metab Res Rev. 2016;32:730-5.

16. Cunningham J, Sprague SM, Cannata-Andia J, et al. Osteoporosis in chronic kidney disease. Am J Kidney Dis. 2004;43:566-71.

17. Hammes H-P, Welp R, Kempe H-P, Wagner C, Siegel E, Holl RW. Risk factors for retinopathy and DME in type 2 diabetes-results from the German/Austrian DPV database. PLoS One. 2015;10:e0132492.

18. Molitich ME, DeFronzo RA, Franz MJ, et al. Nephropathy in diabetes. Diabetes Care. 2004;27: s79-s83.
19. Ritz E, Orth SR. Nephropathy in patients with type 2 diabetes mellitus. N Engl J Med. 1999;341: 1127-33.

20. Boyko EJ, Seelig AD, Ahroni JH. Limb- and personlevel risk factors for lower-limb amputation in the prospective Seattle Diabetic Foot Study. Diabetes Care. 2018;41:891-8.

21. Kidney Disease Statistics for the United States National Institute of Diabetes and Digestive and Kidney Diseases 2016. https://www.niddk.nih.gov/ health-information/health-statistics/kidney-disease. Accessed 20 Feb 2020.

22. Szczech LA, Stewart RC, Su H-L, et al. Primary care detection of chronic kidney disease in adults with type- 2 diabetes: the ADD-CKD study (Awareness, Detection and Drug Therapy in Type 2 Diabetes and Chronic Kidney Disease). PLoS One. 2014;2014: $1-16$.

23. United States Renal Data System. Annual Data Report. Chapter 7: healthcare expenditures for persons with CKD. https://www.usrds.org/2018/ view/v1_07.aspx. Accessed 20 Feb 2020.

24. HHS Launches President Trump's 'Advancing American Kidney Health' Initiative [press release]. U.S. Department of Health and Human Services, July 10, 2019.

25. American Diabetes Association. Standards of medical care in diabetes-2018. Diabetes Care. 2018;41: S1-59.

26. Pálsson R, Patel U. Cardiovascular complications of diabetic kidney disease. Adv Chronic Kidney Dis. 2014;21:273-80.

27. Pan Y, Guo LL, Jin HM. Low-protein diet for diabetic nephropathy: a meta-analysis of randomized controlled trials. Am J Clin Nutr. 2008;88:660-6.

28. DeFronzo RA, Norton L, Abdul-Ghani M. Renal, metabolic and cardiovascular considerations of SGLT2 inhibition. Nat Rev Nephrol. 2017;13:11-26.

29. Brenner BM, Cooper ME, de Zeeuw D, et al. Effects of losartan on renal and cardiovascular outcomes in patients with type 2 diabetes and nephropathy. N Engl J Med. 2001;345:861-9.

30. Lewis EJ, Hunsicker LG, Clarke WR, et al. Renoprotective effect of the angiotensin-receptor antagonist irbesartan in patients with nephropathy due to type 2 diabetes. N Engl J Med. 2001;345:851-60.

31. Mogensen CE, Neldam S, Tikkanen I, et al. Randomised controlled trial of dual blockade of reninangiotensin system in patients with hypertension, microalbuminuria, and non-insulin dependent 
diabetes: the candesartan and lisinopril microalbuminuria (CALM) study. BMJ. 2000;321:1440-4.

32. Dalpoas SE, Samal L, Pham PA. Johns Hopkins Diabetes Guide. Johns Hopkins Medicine 2017. https://www. hopkinsguides.com/hopkins/view/Johns_Hopkins_ Diabetes_Guide/547040/7/Diuretics. Accessed 20 Feb 2020.

33. Mauer M, Zinman B, Gardiner R, et al. Renal and retinal effects of enalapril and losartan in type 1 diabetes. N Engl J Med. 2009;361:40-51.

34. Parving $\mathrm{HH}$, Lehnert $\mathrm{H}$, Brochner-Mortensen J, Gomis R, Andersen S, Arner P. The effect of irbesartan on the development of diabetic nephropathy in patients with type 2 diabetes. $\mathrm{N}$ Engl J Med. 2001;345:870-8.

35. Lewis EJ, Hunsicker LG, Bain RP, Rohde RD. The effect of angiotensin-converting-enzyme inhibition on diabetic nephropathy. N Engl J Med. 1993;329: 1456-62.

36. Ghosh RK, Ghosh SM, Chawla S, Jasdanwala SA. SGLT2 inhibitors: a new emerging therapeutic class in the treatment of type 2 diabetes mellitus. J Clin Pharmacol. 2012;52:457-63.

37. Bays H. Sodium glucose co-transporter type 2 (SGLT2) inhibitors: targeting the kidney to improve glycemic control in diabetes mellitus. Diabetes Ther. 2013;4:195-220.

38. Madhavi K, Samba Reddy D, Kulkarni SK. SGLT2 inhibitors: a new generation of antidiabetic drugs. Int J Pharm Sci Nanotechnol. 2015;8:2787-98.

39. Gerich JE. Role of the kidney in normal glucose homeostasis and in the hyperglycaemia of diabetes mellitus: therapeutic implications. Diabet Med. 2010;27:136-42.

40. Mudaliar S, Polidori D, Zambrowicz B, Henry RR. Sodium-glucose cotransporter inhibitors: effects on renal and intestinal glucose transport: from bench to bedside. Diabetes Care. 2015;38:2344-53.

41. Cherney DZ, Perkins BA, Soleymanlou N, et al. Renal hemodynamic effect of sodium-glucose cotransporter 2 inhibition in patients with type 1 diabetes mellitus. Circulation. 2014;129:587-97.

42. Zinman B, Wanner C, Lachin JM, et al. Empagliflozin, cardiovascular outcomes, and mortality in type 2 diabetes. N Engl J Med. 2015;373:2117-288.

43. Neal B, Perkovic V, Mahaffey KW, et al. Canagliflozin and cardiovascular and renal events in type 2 diabetes. N Engl J Med. 2017;377:644-57.
44. Wiviott SD, Raz I, Bonaca MP, et al. Dapagliflozin and cardiovascular outcomes in type 2 diabetes. N Engl J Med. 2019;380:347-57.

45. Jardine MJ, Mahaffey KW, Neal B, et al. The Canagliflozin and Renal Endpoints in Diabetes with Established Nephropathy Clinical Evaluation (CREDENCE) study rationale and design. Am J Nephrol. 2017;46:462-72.

46. Perkovic V, Jardine MJ, Neal B, et al. Canagliflozin and renal outcomes in type 2 diabetes and nephropathy. N Engl J Med. 2019;380:2295-306.

47. Cannon CP, Perkovic V, Agarwal R, et al. Evaluating the effects of canagliflozin on cardiovascular and renal events in patients with type 2 diabetes and chronic kidney disease according to baseline HbA1c, including those with HbA1c <7\%: results from the CREDENCE trial. Circulation. 2020;141: 407-10.

48. Bakris G, Oshima M, Mahaffey KW, et al. Canagliflozin slows declines in kidney function in people with baseline eGFR $<30 \mathrm{~mL} / \mathrm{min} / 1.73 \mathrm{~m}^{2}$. Presented at: American Society of Nephrology's Kidney Week; November 5-10, 2019; Washington, DC. Poster TH-PO1202.

49. Levin A, Mahaffey KW, Baldassarre J, et al. Renal and cardiovascular outcomes of canagliflozin according to race and ethnicity: a CREDENCE secondary analysis. Presented at: American Society of Nephrology's Kidney Week; November 5-10, 2019; Washington, DC. Poster FR-PO233.

50. Mahaffey KW, Jardine MJ, Bompoint S, et al. Canagliflozin and cardiovascular and renal outcomes in type 2 diabetes and chronic kidney disease in primary and secondary cardiovascular prevention groups. Circulation. 2019;140:739-50.

51. Jardine MJ, Zhou Z, Mahaffey KW, et al. Renal, cardiovascular, and safety outcomes of canagliflozin by baseline kidney function: a secondary analysis of the CREDENCE randomized trial. J Am Soc Nephrol. 2020;31:1128-39.

52. Yamout HM, Perkovic V, Davies M, et al. Efficacy and safety of canagliflozin in patients with type 2 diabetes and stage 3 nephropathy. Am J Nephrol. 2014;40:64-74.

53. Inzucchi SE, Zinman B, Wanner C, et al. SGLT-2 inhibitors and cardiovascular risk: proposed pathways and review of ongoing outcome trials. Diab Vasc Dis Res. 2015;12:90-100.

54. Inzucchi SE, Iliev H, Pfarr E, Zinman B. Empagliflozin and assessment of lower-limb amputations in the EMPA-REG OUTCOME trial. Diabetes Care. 2018;41:e4-e5. 
55. Grunberger G, Camp S, Johnson J, et al. Ertugliflozin in patients with stage 3 chronic kidney disease and type 2 fiabetes mellitus: the VERTIS RENAL Randomized Study. Diabetes Ther. 2018;9:49-66.

56. Muskiet MHA, Tonneijck L, van Bommel EJM, Smits MM, van Raalte DH. Renoprotection in LEADER and EMPA-REG OUTCOME. Lancet Diabetes Endocrinol. 2016;4:812-4.

57. Perrone-Filardi P, Avogaro A, Bonora E, et al. Mechanisms linking empagliflozin to cardiovascular and renal protection. Int J Cardiol. 2017;241: $450-6$.

58. Fioretto P, Zambon A, Rossato M, Busetto L, Vettor R. SGLT2 inhibitors and the diabetic kidney. Diabetes Care. 2016;39:165-71.

59. Weir MR. The kidney and type 2 diabetes mellitus: therapeutic implications of SGLT2 inhibitors. Postgrad Med. 2016;128:290-8.

60. Vallon V, Gerasimova M, Rose MA, et al. SGLT2 inhibitor empagliflozin reduces renal growth and albuminuria in proportion to hyperglycemia and prevents glomerular hyperfiltration in diabetic Akita mice. Am J Physiol Renal Physiol. 2014;306: F194-204.

61. Terami N, Ogawa D, Tachibana H, et al. Long-term treatment with the sodium glucose cotransporter 2 inhibitor, dapagliflozin, ameliorates glucose homeostasis and diabetic nephropathy in $\mathrm{db} / \mathrm{db}$ mice. PLoS One. 2014;9:e100777.

62. Osorio H, Coronel I, Arellano A, et al. Sodium-glucose cotransporter inhibition prevents oxidative stress in the kidney of diabetic rats. Oxid Med Cell Longev. 2012;2012:542042.
63. Woods TC, Satou R, Miyata K, et al. Canagliflozin prevents intrarenal angiotensinogen augmentation and mitigates kidney injury and hypertension in mouse model of type 2 diabetes mellitus. Am J Nephrol. 2019;49:331-42.

64. de Albuquerque RN, Neeland IJ, McCullough PA, Toto RD, McGuire DK. Effects of sodium glucose cotransporter 2 inhibitors on the kidney. Diab Vasc Dis Res. 2018;15:375-86.

65. Zou H, Zhou B, Xu G. SGLT2 inhibitors: a novel choice for the combination therapy in diabetic kidney disease. Cardiovasc Diabetol. 2017;16:1-11.

66. ClinicalTrials.gov. A study to evaluate the effect of dapagliflozin on renal outcomes and cardiovascular mortality in patients with chronic kidney disease (Dapa-CKD). 2018. https://clinicaltrials.gov/ct2/ show/NCT03036150. Accessed 20 Feb 2020.

67. ClinicalTrials.gov. EMPA-KIDNEY (the study of heart and kidney protection with empagliflozin). 2019. https://clinicaltrials.gov/ct2/show/NCT0359 4110. Accessed 20 Feb 2020.

68. INVOKANA (canagliflozin) tablets, for oral use [package insert]. Titusville, NJ: Janssen Pharmaceuticals; September 2019.

69. American Diabetes Association. Standards of medical care in diabetes-2019. Diabetes Care. 2019;42: S7-12.

70. American Diabetes Association. Summary of revisions: standards of medical care in diabetes-2020. Diabetes Care. 2020;43:S4-6. 